\title{
Inhibitory effect of receptor for advanced glycation end product-specific small interfering RNAs on the development of hepatic fibrosis in primary rat hepatic stellate cells
}

\author{
JIN-RONG XIA ${ }^{1}$, TING-TING CHEN ${ }^{2}$, WEI-DONG LI ${ }^{1}$, FENG-LIN LU ${ }^{1}$, \\ JUAN LIU $^{1}$, XIAO-GANG CAI ${ }^{1}$, QIN LU ${ }^{1}$ and CUI-PING YANG ${ }^{3}$ \\ ${ }^{1}$ Department of Gastroenterology, Zhongda Hospital, School of Medicine, Southeast University, Nanjing, Jiangsu 210009; \\ ${ }^{2}$ Department of Gastroenterology, Binzhou People's Hospital, Binzhou, Shandong 256610; \\ ${ }^{3}$ Department of Gastroenterology, Shanghai First People's Hospital, Shanghai 200080, P.R. China
}

Received April 17, 2014; Accepted December 19, 2014

DOI: $10.3892 / \mathrm{mmr} .2015 .3342$

\begin{abstract}
Specific small interfering RNAs (siRNAs) targeting receptor for advanced glycation end products (RAGE) inhibit the expression of RAGE, $\alpha$-smooth muscle actin and type I collagen in the T6 hepatic stellate cells (HSCs), indicating that RAGE is important for the activation of HSCs and the expression of collagen. The present study aimed to investigate the effect of specific siRNAs targeting RAGE on the development of hepatic fibrosis (HF), using primary rat HSCs, which were isolated and cultured in vitro. The expression vectors for specific siRNAs targeting RAGE were constructed and transfected into primary rat HSCs. Untreated and nonspecific siRNA-transfected primary rat HSCs served as controls. The expression levels of RAGE, interleukin-6 (IL-6), tumor necrosis factor- $\alpha$ (TNF- $\alpha$ ), transforming growth factor- $\beta 1$ (TGF- $\beta 1$ ), connective tissue growth factor (CTGF), laminin (LN), hyaluronic acid (HA) and N-terminal procollagen III propeptide (PIIINP) in primary HSCs were detected by reverse transcription quantitative polymerase chain reaction and western blotting. The mRNA and $42 \mathrm{kD}$ protein expression of RAGE in the pAKD-GR126-transfected primary HSCs were significantly downregulated compared with those in the untreated and the pAKD-negative control (NC)-transfected controls. The mRNA and protein expression levels of IL-6, TNF- $\alpha$, TGF- $\beta 1$, CTGF, LN, HA and PIIINP in the pAKD-GR126-transfected primary HSCs were also markedly downregulated compared with those in the untreated and pAKD-NC-transfected controls. Therefore, RAGE-specific
\end{abstract}

Correspondence to: Professor Jin-Rong Xia, Department of Gastroenterology, Zhongda Hospital, School of Medicine, Southeast University, 87 Dingjiaqiao Road, Nanjing, Jiangsu 210009, P.R. China

E-mail: jinrongxiacn@126.com

Key words: primary hepatic stellate cells, hepatic fibrosis, small interfering RNA, receptor for advanced glycation end products
siRNAs inhibited the expression of RAGE in primary rat HSCs and inhibited the development of HF.

\section{Introduction}

Liver cirrhosis is one of the most common illnesses compromising human health and no completely effective clinical treatment exists. The mechanisms underlying the development of hepatic fibrosis (HF) and effective treatments for this condition remain to be elucidated. Receptor for advanced glycation end products (RAGE), a member of the immunoglobulin superfamily (1), is found on the surface of various cell types, including mononuclear macrophages, neurons, renal mesangial cells and vascular endothelial cells. This protein contributes to various diseases, including type 2 diabetes, Alzheimer's disease, chronic kidney disease and atherosclerosis (2-5). AGEs are implicated in the pathogenesis of fibrosis in a number of tissue types $(6,7)$ and evidence indicates that AGEs and RAGE contribute to the pathogenesis of chronic liver disease (6). RAGE can be significantly expressed on several types of liver cell, including hepatic stellate cells (HSCs) (8-10), which are the major effectors during hepatic fibrogenesis (7). The expression of RAGE is also increased in animal models exhibiting chronic liver disease $(11,12)$. Therefore, RAGE may be important in the development of HF and inhibiting RAGE may be a method to prevent or reverse HF.

Specific small interfering RNA (siRNA) targeting RAGE, inhibits the expression levels of RAGE, $\alpha$-smooth muscle actin and collagen type I in T6 HSCs, indicating that RAGE may be important for the activation of HSCs and the expression of collagen (11). The present study aimed to investigate the effect of RAGE-specific siRNAs on the development of $\mathrm{HF}$ in primary rat HSCs.

\section{Materials and methods}

Materials. Three Healthy male Sprague-Dawley rats, aged 15 months and weighing between 400 and $500 \mathrm{~g}$, were purchased from the Nanjing Medical University Laboratory 
Animal Center (Nanjing, China). The study was approved by the Animal Research Ethics Committee of Zhongda Hospital, Southeast University (Nanjing, China). The rats were fed a normal diet and had free access to food and water; in addition, the rats were maintained at a temperature of $22^{\circ} \mathrm{C}$ under a 12-h light/dark cycle. Type IV collagenase, pronase $\mathrm{E}$ and Nycodenz were obtained from Sigma-Aldrich (St. Louis, MO, USA) and DNase I was obtained from Gibco Life Technologies (Carlsbad, CA, USA). The pAKD.CMV.bGlobin.egreen fluorescent protein (GFP).H1.short hairpin (sh)RNA vector was purchased from GenScript USA, Inc. (Piscataway, NJ, USA).

Isolation and culture of primary rat HSCs. HSCs were obtained from rats as previously described (13). Primary rat HSCs were isolated by density gradient centrifugation at $400 \mathrm{x} \mathrm{g}$ for 5 min and cultured with Dulbecco's modified Eagle's medium (DMEM; Sigma-Aldrich) supplemented with $10 \%$ fetal bovine serum in vitro. The cell viability was determined using trypan blue staining (Sigma-Aldrich) and the expression of desmin in cells was detected by immunohistochemistry. The primary HSCs were cultured for 5 days and divided at random into five groups as follows: Cells transfected with pAKD-GR125, pAKD-GR126, pAKD-GR127, pAKD-GR128 or pAKD-GR129.

Preparation of specific siRNAs targeting RAGE. Rat RAGE mRNA (GenBank accession no. NM-053336.1) was used as the target sequence. siRNA Target Finder design software (version 2.0; Ambion, Austin, TX, USA) was used to model the secondary structures of the rat RAGE mRNA and five pairs of 19 nt siRNA sequences were designed in accordance with the target sequences and their complementary sequences (the specificity of the sequences were confirmed using BLAST). These sequences were then converted into short RNA oligonucleotide sequences, which form hairpin structures. $B g l \mathrm{II}$ and $K p n I$ restriction sites and a 9 bp hairpin structure were added to the two ends of the sequence. The final oligonucleotides were named GR125, GR126, GR127, GR128 and GR129 (Table 1).

The $B g l \mathrm{II}$ and $K p n I$ restriction sites and the hairpin structure sequence were also added to the two ends of another pair of non-specific siRNAs (not homologous to rat RAGE mRNA, as confirmed by BLAST). This construct was termed negative control (NC; Table 1).

Construction of specific siRNA expression vectors. The pAKD.CMV.bGlobin.eGFP.H1.shRNA vector was linearized by restriction enzyme digestion using $B g l \mathrm{II}$ and $K p n \mathrm{I}$ and then ligated to the annealed double-stranded DNA fragments GR125, GR126, GR127, GR128 and GR129, forming the RAGE-specific siRNA expression vectors pAKD-GR125, pAKD-GR126, pAKD-GR127, pAKD-GR128 and pAKD-GR129, respectively. The non-specific siRNA expression vector, $\mathrm{pAKD}-\mathrm{NC}$, was constructed in the same way and was used as a control.

Cell transfection. The pAKD-GR125, pAKD-GR126, pAKD-GR127, pAKD-GR128 and pAKD-GR129 RAGE siRNA expression vectors, were transfected into primary rat HSCs individually at multiplicity of infections (MOIs) of 20 , 100, 200 and 1,000. Untreated and nonspecific siRNA-transfected primary rat HSCs were used as controls. The medium was replaced with serum-free DMEM prior to transfection. The total RNA $(0.5 \mu \mathrm{g})$ was extracted and the mRNA expression of RAGE was determined by reverse transcription quantitative polymerase chain reaction (RT-qPCR) following incubation for $48 \mathrm{~h}$ at $37^{\circ} \mathrm{C}(11)$. RT reagent kit was purchased from Takara Bio, Inc. (Dalian, China) and the primers used were as follows: GR-129 sense, 5'-GATCCCCGTGAATCCTGCCTCT GAACTTCAAGAGAGTTCAGAGGCAGGATTCACTTTTT TGTAC-3' and antisense, 5'-AAAAAAGTGAATCCTGC CTCTGAACTCTCTTGAAGTTCAGAGGCAGGATTCAC GGG-3'. ABI-9700 PCR apparatus (Applied Biosystems, Waltham, MA, USA) was used and the cycling parameters were as follows: $94^{\circ} \mathrm{C}$ for $3 \mathrm{~min}$, followed by 40 cycles of $94^{\circ} \mathrm{C}$ for 1 $\min , 55^{\circ} \mathrm{C}$ for $1 \mathrm{~min}$ and $72^{\circ} \mathrm{C}$ for $1 \mathrm{~min}$. $\beta$-actin served as the endogenous control, $\Delta \Delta \mathrm{CT}$ method for relative quantification was used to calculate differences in the expression level of each target gene (14).

The specific siRNA expression vector pAKD-GR126 with the maximum ability to inhibit the expression of RAGE was selected and transfected into primary rat HSCs $\left(0.2 \times 10^{6}\right)$ cultured in DMEM for 5 days at $37^{\circ} \mathrm{C}$. Untreated and nonspecific siRNA-transfected primary rat HSCs served as controls. The cells were collected following incubation for 24, 48 and $72 \mathrm{~h}$, and the total RNA was extracted. The efficiency of RAGE gene silencing was assessed by RT-qPCR, as described below.

Cell treatment. Primary rat HSCs $\left(0.2 \times 10^{6}\right)$ cultured for 5 days at $37^{\circ} \mathrm{C}$ were randomly divided into three groups ( $n=3 /$ group): Group A, blank group, treated with AGE-bovine serum albumen (BSA, $200 \mathrm{mg} / \mathrm{l}$ ); the pAKD-GR126 group, treated with AGE-BSA (200 mg/l) and pAKD-GR126 (MOI=1,000) and the pAKD-NC group, treated with AGE-BSA (200 mg/l) and pAKD-NC (MOI=1,000). Each group had three repeats. The medium was replaced with serum-free DMEM prior to transfection.

Total RNA extraction and RT-qPCR assay. The total RNA was extracted from the primary rat HSCs using TRIzol reagent (Sigma-Aldrich) according to the manufacturer's instructions. The purity and concentration of the RNA were determined prior to the RNA being reverse transcribed, as previously described (11). The cycling parameters were as follows: $94^{\circ} \mathrm{C}$ for $3 \mathrm{~min}$ and 40 cycles of $94^{\circ} \mathrm{C}$ for $1 \mathrm{~min}, 55^{\circ} \mathrm{C}$ for $1 \mathrm{~min}$ and $72^{\circ} \mathrm{C}$ for $1 \mathrm{~min}$. $\beta$-actin served as an internal control. The $2^{-\Delta \Delta \mathrm{CT}}$ method for relative quantification was used to calculate the differences in the expression level of each target gene $(11,14)$.

Western blot analysis. The cellular proteins were extracted using modified radioimmunoprecipitation buffer (Sigma-Aldrich) containing 50 mM Tris- $\mathrm{HCl}$ (pH 7.5), 150 mM NaCl, 1\% NP-40, $0.1 \%$ sodium dodecyl sulphate (SDS), $0.5 \%$ deoxycholate, $1 \mathrm{mM}$ EDTA, $2 \mathrm{mg} / \mathrm{l}$ leupeptin and $1 \mathrm{mM}$ phenylmethylsulfonyl fluoride. The protein concentration was determined using a bicinchoninic acid assay. The proteins $(50 \mu \mathrm{g})$ were separated by electrophoresis on a $10 \%$ gradient SDS-polyacrylamide gel (Sigma-Aldrich) and transferred onto polyvinylidene fluoride membranes (Sigma-Aldrich). Following protein transfer, the membranes were blocked with 5\% non-fat milk (Sigma-Aldrich) in Tris-buffered saline containing $0.1 \%$ Tween-20 (Sigma-Aldrich) for $1 \mathrm{~h}$ at room temperature and then incubated 
Table I. Sequences of specific small interfering RNAs targeting receptor for advanced glycation end products.

\begin{tabular}{ll}
\hline Oligonucleotide & Sequence \\
\hline GR125 & \\
Sense & 5'-GATCCCCGCCAACCCAGAAGCTAGAATTCAAGAGATTCTAGCTTCTGGGTTGGCTTTTTTGTAC-3' \\
Antisense & 5'-AAAAAAGCCAACCCAGAAGCTAGAATCTCTTGAATTCTAGCTTCTGGGTTGGCGGG-3' \\
GR126 & \\
Sense & 5'-GATCCCCGTGAATCCTGCCTCTGAACTTCAAGAGAGTTCAGAGGCAGGATTCACTTTTTTGTAC-3' \\
Antisense & 5'-AAAAAAGTGAATCCTGCCTCTGAACTCTCTTGAAGTTCAGAGGCAGGATTCACGGG-3' \\
GR127 & \\
Sense & 5'-GATCCCCGCCTCTGAACTCACAGCCATTCAAGAGATGGCTGTGAGTTCAGAGGCTTTTTTGTAC-3' \\
Antisense & 5'-AAAAAAGCCTCTGAACTCACAGCCATCTCTTGAATGGCTGTGAGTTCAGAGGCGGG-3' \\
GR128 & \\
Sense & 5'-GATCCCCGAAGGTGGAACAGTCGCTCTTCAAGAGAGAGCGACTGTTCCACCTTCTTTTTTGTAC-3' \\
Antisense & 5'-AAAAAAGAAGGTGGAACAGTCGCTCTCTCTTGAAGAGCGACTGTTCCACCTTCGGG-3' \\
GR129 & \\
Sense & 5'-GATCCCCGCGAAAACGACAACCCAGATTCAAGAGATCTGGGTTGTCGTTTTCGCTTTTTTGTAC-3' \\
Antisense & 5'-AAAAAAGCGAAAACGACAACCCAGATCTCTTGAATCTGGGTTGTCGTTTTCGCGGG-3' \\
NC & \\
Sense & 5'-GATCCCCTTCTCCGAACGTGTCACGTTTCAAGAGAACGTGACACGTTCGGAGAATTTTTTGATC-3' \\
Antisense & 5'-AAAAAATTCTCCGAACGTGTCACGTTCTCTTGAAACGTGACACGTTCGGAGAAGGG-3' \\
\hline
\end{tabular}

NC, negative control.

A

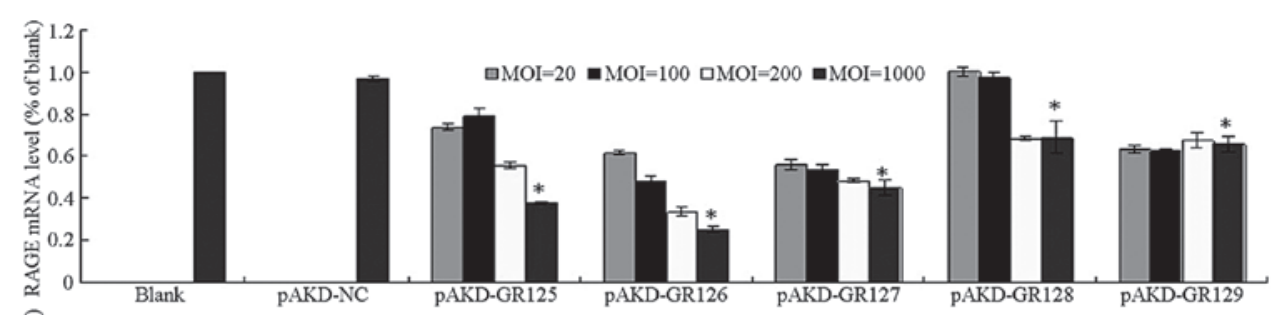

B

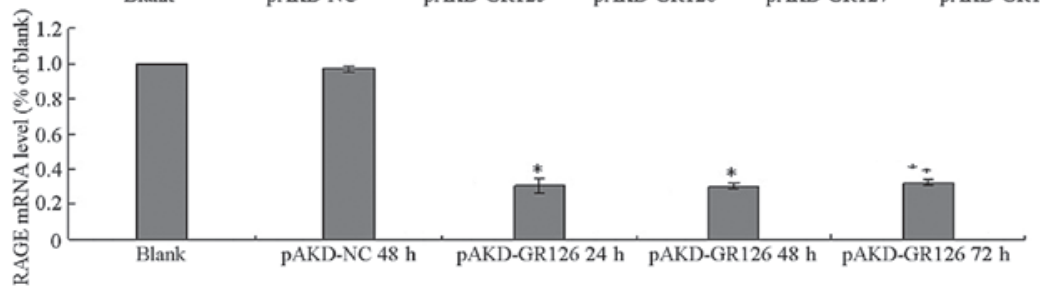

Figure 1. Inhibitory effect of RAGE-specific siRNAs on the expression of RAGE in primary rat HSCs. The mRNA expression of RAGE is presented as the ratio relative to the mRNA expression of $\beta$-actin. The mRNA expression levels were determined by reverse transcription quantitative polymerase chain reaction and the changes are expressed relative to that of $\beta$-actin (blank). (A) Effect of RAGE-specific siRNAs on the mRNA expression of RAGE in primary rat HSCs at different doses ("P $<0.05$, vs. blank and pAKD-NC). (B) Effect of RAGE-specific siRNAs on the mRNA expression of RAGE in primary rat HSCs at various time-points. ( $\mathrm{P}<0.05$, vs. blank and pAKD-NC at $48 \mathrm{~h}$ ). MOI, multiplicity of infection; RAGE, receptor for advanced glycation end products; si, small interfering; NC, negative control; HSC, hepatic stellate cell; blank, untransfected cells.

overnight at $4^{\circ} \mathrm{C}$ with the following primary antibodies: Rabbit anti-rat polyclonal RAGE (ab3611), rabbit anti-rat polyclonal interleukin (IL)-6 (ab6672), mouse anti-rat monoclonal tumor necrosis factor (TNF)- $\alpha$ (ab92324), mouse anti-rat monoclonal transforming growth factor (TGF)- $\beta 1$ (ab64715), rabbit anti-rat polyclonal connective tissue growth factor (CTGF; ab6992), mouse anti-rat monoclonal laminin (LN; ab8983), rabbit anti-rat polyclonal hyaluronic acid (HA; ab20084), mouse anti-rat polyclonal N-terminal procollagen III propeptide (PIIINP; ab169636) and mouse anti-rat monoclonal $\beta$-actin (ab8226), which were all purchased from Abcam (Cambridge, MA, USA). The membranes were subsequently washed with TBST and then exposed to a secondary horseradish peroxidase-labelled antibody [ab6721, goat polyclonal antibodies against rabbit IgG - H\&L (HRP) and ab193651, rabbit polyclonal antibodies against mouse IgG - H\&L (HRP)] in the blocking solution for $1 \mathrm{~h}$ at room temperature. The band intensities were measured using an enhanced chemiluminescent reagent (Western-Lightening Plus; PerkinElmer Life Sciences, Waltham, MA, USA) and the protein signals were normalized against $\beta$-actin. 
A

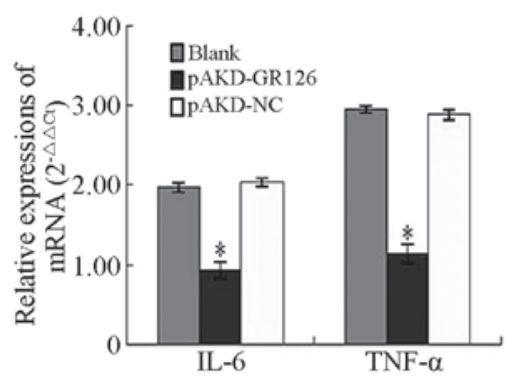

B

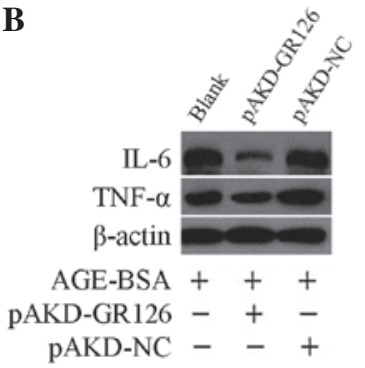

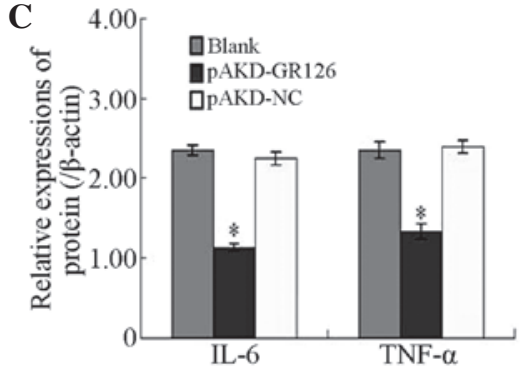

Figure 2. Inhibitory effect of pAKD-GR126 on the mRNA and protein expression levels of the IL- 6 and TNF- $\alpha$ pro-inflammatory cytokines in primary HSCs. (A) mRNA expression levels of IL-6 and TNF- $\alpha$ were determined by reverse transcription quantitative polymerase chain reaction. (B) Protein expression levels of IL- 6 and TNF- $\alpha$ were determined by western blot analysis. (C) Protein expression levels of IL- 6 and TNF- $\alpha$ are expressed as ratios relative to the expression of $\beta$-actin and were determined using densitometric scanning ( $\mathrm{P}<0.05$, vs. blank and pAKD-NC). IL, interleukin; TNF, tumor necrosis factor; NC, negative control; BSA, bovine serum albumen; Blank, untransfected cells; $\mathrm{Ct}$, cycle threshold.

$\mathbf{A}$

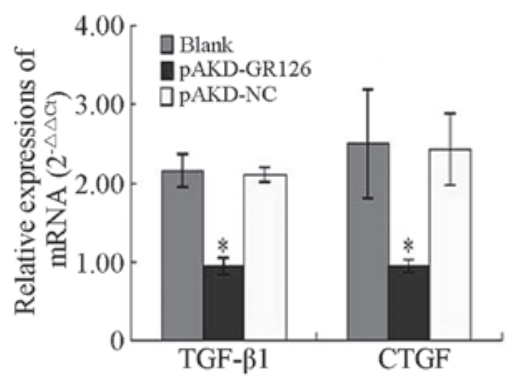

B

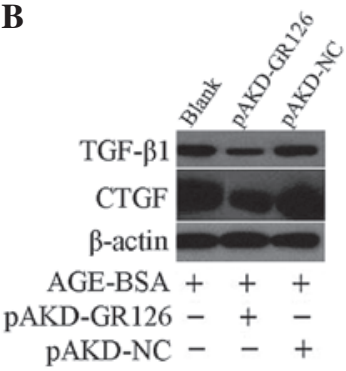

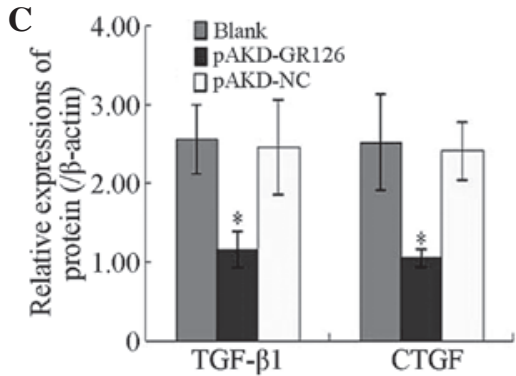

Figure 3. Inhibitory effect of pAKD-GR126 on the mRNA and protein expression levels of the TGF- $\beta 1$ and CTGF profibrogenic cytokines in primary HSCs. (A) mRNA expression levels of TGF- $\beta 1$ and CTGF were determined by reverse transcription quantitative polymerase chain reaction. (B) Protein expression levels of TGF- $\beta 1$ and CTGF were determined by western blot analysis. (C) Protein expression levels of TGF- $\beta 1$ and CTGF are expressed as ratios relative to the protein expression of $\beta$-actin and were determined by densitometric scanning ("P<0.05, vs. blank and pAKD-NC). TGF, transforming growth factor; CTGF, connective tissue growth factor; HSC, hepatic stellate cell; BSA, bovine serum albumin; Blank, untransfected cells; Ct, cycle threshold; NC, negative control.
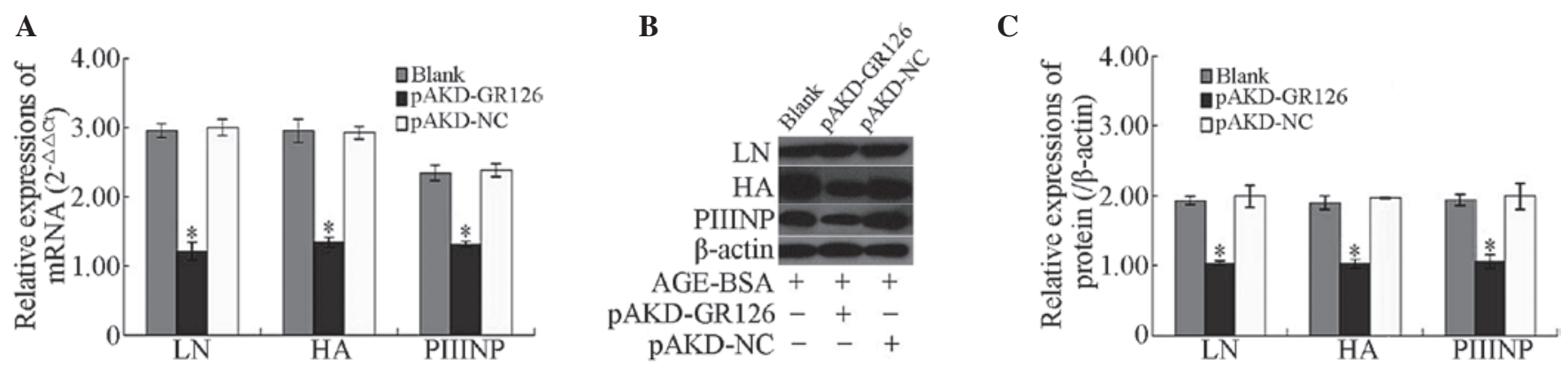

Figure 4. Inhibitory effect of pAKD-GR126 on the mRNA and protein expression levels of the LN, HA and PIINP fibrosis markers in primary HSCs (A) mRNA expression levels of LN, HA and PIIINP were determined by reverse transcription quantitative polymerase chain reaction. (B) Protein expression levels of LN, HA and PIIINP were determined by western blot analysis. (C) Protein expression levels of LN, HA and PIIINP are expressed as ratios relative to the protein expression of $\beta$-actin and were determined by densitometric scanning ("P<0.05, vs. blank and pAKD-NC). LN, laminin; HA, hyaluronic acid; PIIINP, procollagen III propeptide; NC, control; BSA, bovine serum albumen; HSC, hepatic stem cell; Blank, untransfected cells; Ct, cycle threshold.

Statistical analysis. Statistical analyses were performed using SPSS 13.0 statistical software (SPSS, Inc., Chicago, IL, USA). The data were analyzed using one-way analysis of variance and Student-Newman-Keuls multiple comparison test. $\mathrm{P}<0.05$ was considered to indicate a statistically significant difference. The data are expressed as the mean \pm standard deviation.

\section{Results}

Inhibitory effect of RAGE-specific siRNAs on the expression of RAGE in primary rat HSCs. The mRNA expression of RAGE was significantly downregulated in the primary rat HSCs treated with pAKD-GR125, pAKD-GR126, pAKD-GR127, pAKD-GR128 and pAKD-GR129 (all $\mathrm{P}<0.05$ ) compared with the untreated primary rat HSCs and the HSCs treated with pAKD-NC. The mRNA expression of RAGE decreased in a dose-dependent manner in the MOI range of between 20 and 1,000. The most marked decrease was observed in the HSCs treated with pAKD-GR126 at an MOI of 1,000 (Fig. 1A). The mRNA expression of RAGE was downregulated in the primary rat HSCs treated with pAKD-GR126 at 24, 48 and $72 \mathrm{~h}($ all $\mathrm{P}<0.05)$ compared with the untreated cells. However, inhibition in the mRNA expression of RAGE exhibited no differences between 24 and $72 \mathrm{~h}$ 
(P>0.05; Fig. 1B). These results indicated that the RAGE siRNA expressed by pAKD-GR126 effectively inhibited the expression of RAGE.

Inhibitory effect of pAKD-GR126 on the mRNA and protein expression levels of the IL- 6 and TNF- $\alpha$ pro-inflammatory cytokines in primary HSCs. The mRNA and protein expression levels of the IL- 6 and TNF- $\alpha$ pro-inflammatory cytokines, were significantly downregulated in the primary rat HSCs treated with pAKD-GR126 $(\mathrm{P}<0.05)$ compared with the untreated primary rat HSCs and those treated with the pAKD-NC (Fig. 2).

Inhibitory effect of $p A K D-G R 126$ on the mRNA and protein expression levels of the TGF- $\beta 1$ and CTGF profibrogenic cytokines in primary HSCs. The mRNA and protein expression levels of the TGF- $\beta 1$ and CTGF profibrogenic cytokines were significantly downregulated in the primary rat HSCs treated with pAKD-GR126 $(\mathrm{P}<0.05)$, compared with the untreated primary rat HSCs and those treated with the pAKD-NC (Fig. 3).

Inhibitory effect of $p A K D-G R 126$ on the $m R N A$ and protein expression levels of the LN, HA and PIIINP fibrosis markers in primary HSCs. The mRNA and protein expression levels of the LN, HA and PIIINP fibrosis markers were significantly downregulated in the primary rat HSCs treated with pAKD-GR126 $(\mathrm{P}<0.05)$, compared with the untreated primary rat HSCs and those treated with the pAKD-NC (Fig. 4).

\section{Discussion}

The present study demonstrated for the first time, to the best of our knowledge, that specific siRNAs inhibited the effect of RAGE on the development of HF in primary rat HSCs. Increasing investigations are being performed on the biological effects of AGEs and their receptors (15-18) and the role of the AGE-RAGE axis as a cofactor in the development of liver fibrosis (15-18). Honsawek et al revealed that serum RAGE is associated with the severity of biliary atresia and, therefore, may serve as an indicator reflecting the severity and development of HF in individuals with postoperative biliary atresia (19). Goodwin et al demonstrated that AGEs were damaging to the liver and augmented HF in an animal model exhibiting chronic liver disease, and these effects of which were associated with the activation of myofibroblasts (20). The upregulation of RAGE, induced by AGE administration, may be important in mediating these effects. Additionally, the serum AGE levels are significantly increased in patients with liver cirrhosis (21-23). RAGE is exclusively expressed in HSCs and myofibroblasts in the rat liver and its expression is upregulated during $\mathrm{HSC}$ activation and transdifferentiation into myofibroblasts (24), during which, the expression of TGF- $\beta 1$ is significantly increased (8). These previous findings suggest that RAGE may be a major receptor involved in the activation and transdifferentiation of HSCs into myofibroblasts and that the expression of RAGE in the liver is important in liver fibrosis.

Several methods to inhibit RAGE in liver cells have been developed. Lin et al revealed that curcumin can suppress the gene expression of RAGE by increasing the activity of PPAR $\gamma$ and attenuating oxidative stress, leading to the elimination of the effects of AGEs on the activation of HSCs (25). Our previous study demonstrated that a specific siRNA targeting RAGE inhibited HF in a rat model (5). The present study constructed expression vectors for specific siRNAs targeting RAGE and transfected these into primary rat HSCs. The results revealed that the mRNA expression of RAGE was downregulated in the primary rat HSCs treated with pAKD-GR126 compared with the untreated primary rat HSCs and those treated with pAKD-NC. These results indicated that the RAGE-specific siRNA expressed by pAKD-GR126 effectively inhibited the gene expression of RAGE.

Furthermore, the activation of HSCs is important in HF (26-30) and several cytokines are important in the activation of HSCs (31-34). Pro-inflammatory cytokines, including IL- 6 and TNF- $\alpha$, promote the activity and proliferation of HSCs $(35,36)$. TGF- $\beta 1$ and its downstream target, CTGF, are potent activators of HSCs and important profibrogenic cytokines (37-40). These cytokines promote the activation and transdifferentiation of HSCs into myofibroblasts (41) and promote the synthesis and secretion of extracellular matrix (ECM) components $(42,43)$. The ECM and its degradation products, including LN, HA and PIIINP, are useful fibrosis markers and the expression of these products are closely associated with the degree of HF (18). The present study indicated that the mRNA and protein expression levels of RAGE, IL-6, TNF- $\alpha$, TGF- $\beta 1$, CTGF, LN, HA and PIIINP were downregulated in primary rat HSCs treated with pAKD-GR126 compared with the untreated primary rat HSCs and those treated with pAKD-NC. These results demonstrated that the RAGE-specific siRNA expressed by pAKD-GR126 effectively inhibited gene expression of RAGE and also inhibited the expression levels of IL- 6 , TNF- $\alpha$, TGF- $\beta 1$, CTGF, LN, HA and PIIINP. Although the effect of RAGE on the development of HF remains to be fully elucidated, these findings suggested that RAGE may be a novel target for treating liver fibrosis and that RAGE-specific siRNA may be an effective candidate for the prevention of liver fibrogenesis.

In conclusion, the present study revealed for the first time, to the best of our knowledge, that a RAGE-specific siRNA can inhibit the effect of RAGE on the development of HF in primary rat HSCs. The results demonstrated that the mRNA and protein expression levels of pro-inflammatory cytokines, profibrogenic cytokines and fibrosis markers were significantly downregulated in cells treated with RAGE-specific siRNA, indicating that RAGE may be a novel target for treatment of liver fibrosis, and that RAGE-specific siRNA may be an effective candidate to prevent liver fibrogenesis. However, there were limitations to the present study, including the number of control groups and the lack of repeats, therefore, the results obtained are not sufficient to provide a firm conclusion.

\section{Acknowledgements}

The authors would like to thank Dr Changqing Yang of the Department of Gastroenterology, Tongji Hospital of Tongji University School of Medicine (Tongji, China) for their technical assistance. This study was supported by the Natural Science Foundation of Jiangsu Province (no. BK2009284). 


\section{References}

1. Bierhaus A, Humpert PM, Morcos M, et al: Nawroth, Understanding RAGE, the receptor for advanced glycation end products. J Mol Med (Berl) 83: 876-886, 2005.

2. Hegab Z, Gibbons S, Neyses L and Mamas MA: Role of advanced glycation end products in cardiovascular disease. World J Cardiol 4: 90-102, 2012

3. Yan SD, Bierhaus A, Nawroth PP and Stern DM: RAGE and Alzheimer's disease: a progression factorfor amyloid-beta-induced cellular perturbation? J Alzheimers Dis 16: 833-843, 2009.

4. Oleniuc M, Secara I, Onofriescu M, Hogas S, et al: Consequences of advanced glycation end products accumulation in chronic kidney disease and clinical usefulness of their assessment using a non-invasive technique-skin autofluorescence. Maedica (Buchar) 6: 298-307, 2011.

5. Barlovic DP, Soro-Paavonen A and Jandeleit-Dahm KA: RAGE biology, atherosclerosis and diabetes. Clin Sci (Lond) 211: 43-55, 2011.

6. Schwenger V, Morath C, Salava A, et al: Damage to the peritoneal membrane by glucose degradation products is mediated by the receptor for advanced glycation end-products. J Am Soc Nephrol 17: 199-207, 2006.

7. Vlassara H, Striker LJ, Teichberg S, et al: Advanced glycation end products induce glomerular sclerosis and albuminuria in normal rats Proc Natl Acad Sci USA 91: 11704-11708, 1994.

8. Fehrenbach H, Weiskirchen R, Kasper M and Gressner AM: Up-regulated expression of the receptor for advanced glycation end products in cultured rat hepatic stellate cells during transdifferentiation to myofibroblasts. Hepatology 34: 943-952, 2001.

9. Guimarães EL, Empsen C, Geerts A and van Grunsven LA: Advanced glycation end products induce production of reactive oxygen species via the activation of NADPH oxidase in murine hepatic stellate cells. J Hepatol 52: 389-397, 2010.

10. Iwamoto K, Kanno K, Hyogo H, et al: Advanced glycation end products enhance the proliferation and activation of hepatic stellate cells. J Gastroenterol 43: 298-304, 2008

11. Xia JR, Liu NF and Zhu NX: Specific siRNA targeting the receptor for advanced glycation end products inhibits experimental hepatic fibrosis in rats. Int J Mol Sci 9: 638-661, 2008.

12. Lohwasser C, Neureiter D, Popov Y, Bauer M and Schuppan D: Role of the receptor for advanced glycation end products in hepatic fibrosis. World J Gastroenterol 15: 5789-5798, 2009.

13. Friedman SL and Roll FJ: Isolation and culture of hepatic lipocytes, Kuper cells, and sinusoidal endothelial cells by density gradient centreifugation with Stractan. Anal Biochem 161: 207-218, 1987.

14. Livak KJ and Schmittgen TD: Analysis of relative gene expression data using real-time quantitative PRC and the 2 (- Delta Delta C(T) method. Methods 25: 402-408, 2001.

15. Xie X, Zhao R and Shen GX: Impact of cyanidin-3-glucoside on glycated LDL-induced NADPH oxidase activation, mitochondrial dysfunction and cell viability in cultured vascular endothelial cells. Int J Mol Sci 13: 15867-15880, 2012

16. Sangle GV, Zhao R, Mizuno TM and Shen GX: Involvement of RAGE, NADPH oxidase, and Ras/Raf-1 pathway in glycated LDL-induced expression of heat shock factor-1 and plasminogen activator inhibitor-1 in vascular endothelial cells. Endocrinology 151: 4455-4466, 2010.

17. Willems S, Verleden SE, Vanaudenaerde BM, et al: Multiplex protein profiling of bronchoalveolar lavage in idiopathic pulmonary fibrosis and hypersensitivity pneumonitis. Ann Thorac Med 8: 38-45, 2013.

18. Cheng A, Dong Y, Zhu F, et al: AGE-LDL activates Toll like receptor 4 pathway and promotes inflammatory cytokines production in renal tubular epithelial cells. Int J Biol Sci 9: 94-107, 2013

19. Honsawek S, Vejchapipat P, Payungporn S, et al: Soluble receptor for advanced glycation end products and liver stiffness in postoperative biliary atresia. Clin Biochem 46: 214-218, 2013.

20. Goodwin M, Herath C, Jia Z, et al: Advanced glycation end products augment experimental hepatic fibrosis. J Gastroenterol Hepatol 28: 369-376, 2013

21. Sebeková K, Kupcová V, Schinzel R and Heidland A: Markedly elevated levels of plasma advanced glycation end products in patients with liver cirrhosis-amelioration by liver transplantation. J Hepatol 36: 66-71, 2002.
22. Ahmed N, Lüthen R, Häussinger D, et al: Increased protein glycation in cirrhosis and therapeutic strategies to prevent it. Ann N Y Acad Sci 1043: 718-724, 2005.

23. Yagmur E, Tacke F, Weiss C, et al: Elevation of Nepsilon-(carboxymethyl) lysine-modified advanced glycation end products in chronic liver disease is an indicator of liver cirrhosis. Clin Biochem 39: 39-45, 2006.

24. Oldfield MD, Bach LA, Forbes JM, et al: Advanced glycation end products cause epithelial-myofibroblast transdifferentiation via the receptor for advanced glycation end products (RAGE). J Clin Invest 108: 1853-1863, 2001.

25. Lin J, Tang Y, Kang Q, Feng Y and Chen A: Curcumin inhibits gene expression of receptor for advanced glycation end-products (RAGE) in hepatic stellate cells in vitro by elevating PPAR $\gamma$ activity and attenuating oxidative stress. Br J Pharmacol 166: 2212-2227, 2012.

26. Reynaert H, Thompson MG, Thomas T and Geerts A: Hepatic stellate cells: role in microcirculation and pathophysiology of portal hypertension. Gut 50: 571-581, 2002.

27. Shi GF and Li Q: Effects of oxymatrine on experimental hepatic fibrosis and its mechanism in vivo. World J Gastroenterol 11: 268-271, 2005.

28. Reeves HL and Friedman SL: Activation of hepatic stellate ceils-a key issue in liver fibrosis. Front Biosci 7: d808-d826, 2002.

29. Campbell JS, Hughes SD, Gilbertson DG, et al: Pletelet-derived growth factor $\mathrm{C}$ induces liver fibrosis, steatosis, and hepatocellular carcinoma. Proc Natl Acad Sci USA 102: 3389-3394, 2005.

30. Sakaida I, Hironaka K, Kimura T, et al: Herbal medicine sho-saiko-to(TJ-9) increases expression matrix metalloproteinases (MMPs) with reduced expression of tissue inhibitor of metalloproteinases (TIMPs) in rat stellate cell. Life Sci 74: 2251-2263, 2004

31. Iredale JP: Hepatic stellate cell behavior during resolution of liver injury. Semin Liver Dis 21: 427-436, 2001

32. Benyon RC and Arthur MJ: Extracellular matrix degradation and the role of hepatic stellate cells. Semin Liver Dis 21: 373-384, 2001.

33. Borkham-Kamphorst E, Herrmann J, Stoll D, et al: Dominent-negative solute PDGF-beta receptor inhibits hepatic stellate cell activation and attenuates liver fibrosis. Lab Invest 84 766-777, 2004.

34. Novosyadlyy R, Tron K, Dudas J, Ramadori G and Scharf JG: Expression and regulation of the insulin-like growth factor axis components in rat liver myofibroblasts. J Cell Physiol 199: 388-398, 2004

35. Simeonova PP, Gallucci RM, Hulderman T, et al: The role of tumor necrosis factor-alpha in liver toxicity, inflammation, and fibrosis induced by carbon tetrachloride. Toxicol Appl Pharmacol 177: 112-120, 2001.

36. da Silva FM, Guimarães EL, Grivicich I, et al: Hepatic stellate cell activation in vitro: cell cycle arrest at $\mathrm{G} 2 / \mathrm{M}$ and modification of cell motility. J Cell Biochem 90: 387-396, 2003.

37. Huang $G$ and Brigstock DR: Integrin expression and function in the response of primary culture hepatic stellate cells to connective tissue growth factor (CCN2). J Cell Mol Med 15: 1087-1095, 2011.

38. Galicia-Moreno M, Rodríguez-Rivera A, Reyes-Gordillo K, et al: Trolox down-regulates transforming growth factor-beta and prevents experimental cirrhosis. Basic Clin Pharmacol Toxicol 103: 476-481, 2008.

39. Chen A: Acetaldehyde stimulates the activation of latent transforming growth factor-betal and induces expression of the type II receptor of the cytokine in rat cultured hepatic stellate cells. Biochem J 368: 683-693, 2002

40. Sedlaczek N, Jia JD, Bauer M, et al: Proliferating bile duct epithelial cells are a major source of connective tissue growth factor in rat bilinry fibrosis. Am J Pathol 158: 1239-1244, 2001.

41. Faouzi S,Le Bail B, Neaud V, et al: Myofibroblasts are responsible for collagen synthesis in the stroma of human hepatocellular carcinoma: an in vivo and in vitro study. J Hepatol 30: 275-284, 1999.

42. Friedman SL: Molecular regulation of hepatic fibrosis, an integrated cellular response to tissue injury. J Biol Chem 275: 2247-2250, 2000

43. Pinzani M and Marra F: Cytokine receptors and signaling in hepatic stellate cells. Semin Liver Dis 21: 397-416, 2001. 